\title{
Improvement in Efficiency and Operating Range of Centrifugal Blower Stage for Sewage Aeration Blower
}

\author{
Kiyotaka Hiradate $^{1}$, Toshio Kanno ${ }^{2}$, Hideo Nishida ${ }^{2}$, \\ Yasushi Shinkawa ${ }^{2}$ and Satoshi Joukou ${ }^{2}$ \\ ${ }^{1}$ Mechanical Engineering Research Laboratory, Hitachi, Ltd. \\ 832-2, Horiguchi, Hitachinaka, Ibaraki 312-0034, Japan, kiyotaka.hiradate.kf@hitachi.com \\ ${ }^{2}$ Hitachi Plant Technology, Ltd. \\ 603, Kandatsu, Tsuchiura, Ibaraki 300-0013, Japan
}

\begin{abstract}
We developed a high-efficiency, wide-operating-range centrifugal blower stage to meet the demand for reduced total energy-consumption in sewage treatment plants. We improved the efficiency of the two-dimensional impeller using a shape optimization tool and one-dimensional performance prediction tool. A limit of the throat deceleration ratio was set to maintain the stall-margin of the impeller. The low solidity vaned diffuser and return channel were designed using a sensitivity analysis with orthogonal arrays and three-dimensional steady flow simulations. The low solidity diffuser was designed in order to improve the performance in the low-flow-rate region. The return channel was designed so that the total pressure loss in the return channel was minimized. Model tests of both the conventional and optimized blower stages were carried out, and the efficiency and operating range of both stages were compared. The optimized blower stage improved in stage efficiency by $3 \%$ and in operating range by $5 \%$ compared with the conventional blower stage.
\end{abstract}

Keywords: Centrifugal Blower, Orthogonal Array, Numerical Simulation, Efficiency, Operating Range

\section{Introduction}

Sewage discharged from homes or factories is transferred to sewage treatment plant through sewers. At the sewage treatment plant, pre-treatment is first carried out to remove relatively large particles from the sewage. Then, the sewage is carried to a reaction tank in which bacteria decomposing organic materials in the sewage exist. These bacteria require oxygen to decompose organic matter, so air is supplied to the sewage in a process called aeration. Sewage-aeration blowers are utilized to supply oxygen to bacteria in the sewage.

From the viewpoint of conservation of the global environment, demands to reduce the total energy-consumption in sewage treatment plants have risen year by year. The sewage-aeration blower accounts for approximately 50 percent of total energy consumption in the plant. Consequently, the demand is increasing to improve the efficiency of sewage-aeration blowers to reduce energy consumption in these plants. In addition, the amount of sewage varies depending on the time of day and the season of the year. The amount of air used in the aeration greatly varies, too. Therefore, a wide operating range is necessary for sewage aeration blower.

To meet these needs, we have developed a high-performance blower stage. We describe here the design features of our new blower stage and the results of a single-stage model test. In this study, we focused on developing a new blower stage of suction flow coefficient at the design flow rate $\phi_{1, d e s}=0.063$.

\section{Structure of Sewage-Aeration Blower}

Figure 1 shows a schematic diagram of a conventional sewage aeration blower. As shown in the figure, the blower consists of a suction nozzle, inlet guide vanes (IGVs), some centrifugal blower stages that contain a closed centrifugal impeller, low solidity vaned diffuser (Senoo, et al. [1], Hayami, et al. [2] and Sakaguchi, et al. [3]) and return channel, and a scroll. The impeller blades and stator vanes have two-dimensional shapes with a constant thickness. The outlet diameter of the impeller, $D_{2}$, was 925 $\mathrm{mm}$, and the impeller rotational speed was $3550 \mathrm{~min}^{-1}$. 


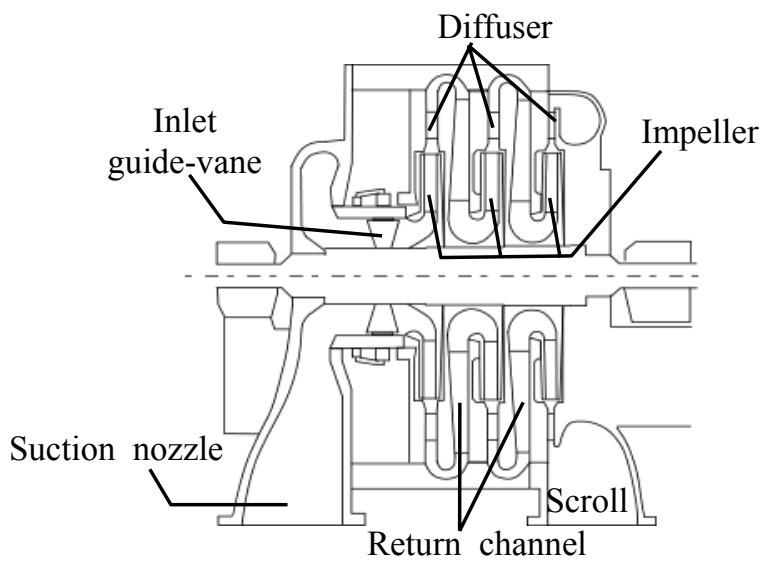

Fig. 1 Schematic diagram of conventional sewage-aeration blower

\section{Design of High-Performance Blower Stage}

\subsection{Impeller Design}

Just as with the conventional impeller, we applied two-dimensional blades to a new impeller. We optimized the twodimensional impeller using a shape-optimization tool (Tanaka, et al. [4]). This tool defines the impeller shapes by changing combinations of the values of the design variables in the impeller and conducts the performance predictions of the blower stages that contain these impellers. Results of the performance predictions were output in decreasing order of stage efficiency. With this tool, we can optimize the distributions of the blade angle, which are defined as the functions of the radius, the blade heights at the impeller inlet and outlet, and the number of blades.

In the blade shape optimization, the shroud shape was changed from a straight line used in the conventional impeller to a onecircular arc. In addition, we used a different method of defining the blade camber line. A one-circular camber line was used in the conventional impeller. On the contrary, a blade angle distribution defined as the quadratic curve of the radius was used in the blade optimization.

To evaluate an effect of the degree of reaction, $\Lambda$, on a total pressure loss in a diffuser and a return channel, performance predictions were conducted for all blower stages that were composed of the impellers defined by the above method, a vaneless diffuser and an outlet component that had equal total pressure loss coefficient, $\zeta_{r c}$, to that of the conventional return channel. In the performance predictions, we used a one-dimensional performance prediction tool (Mishina, et al. [5]). In this tool, the conditions of the flow and the total pressure loss in each part of the blower stage are predicted by some simple one-dimensional equations and empirical coefficients.

One effective way to improve the impeller efficiency is to increase the deceleration ratio, $w_{1} / w_{2}$, and the impeller incidence, $i_{1}$. However, an optimization that is achieved only by improving the impeller efficiency may lead to selecting impellers with a narrow operating range. Therefore, a limit to the throat deceleration ratio, $w_{l} / w_{t h r}$, which was supposed to have a large effect on the stall margin of the impeller, was established. Then the impeller optimization was conducted so that $w_{l} / w_{t h r}$ did not exceed the limit.

The blade shape optimization was conducted under the conditions described above. Table 1 compares the design results between the conventional and optimized impellers. In addition, Fig. 2 compares the meridional shapes and the blade angle distributions between both impellers.

Table 1 Comparison of design results of impeller

\begin{tabular}{|c|c|c|c|}
\hline & & Conventional & Optimized \\
\hline Number of blades & $Z_{i m p}$ & 19 & 15 \\
\hline Outlet blade angle & $\beta_{2 b}$ & $44^{\circ}$ & $45^{\circ}$ \\
\hline Shroud shape & - & Straight-line & One-circular \\
\hline Throat area & $A_{t h r}$ & $69146 \mathrm{~mm}^{2}$ & $68346 \mathrm{~mm}^{2}$ \\
\hline Deceleration ratio & $w_{l} / w_{2}$ & 1.13 & 1.17 \\
\hline Incidence & $i_{l}$ & $-3.4^{\circ}$ & $-2.1^{\circ}$ \\
\hline Throat Deceleration ratio & $w_{l} / w_{t h r}$ & 1.07 & 1.05 \\
\hline Degree of reaction & $\Lambda$ & 0.59 & 0.63 \\
\hline Impeller efficiency improvement & $\Delta \eta_{i m p}$ & - & $+1.1 \%$ \\
\hline Stage efficiency improvement & $\Delta \eta_{a d}$ & - & $+2.1 \%$ \\
\hline
\end{tabular}


Conventional impeller

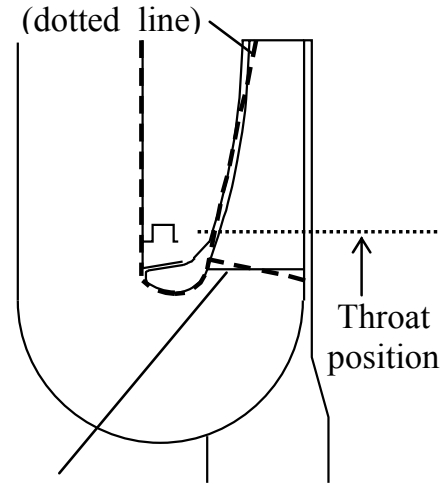

New impeller (continuous line)

(a) Meridional shape

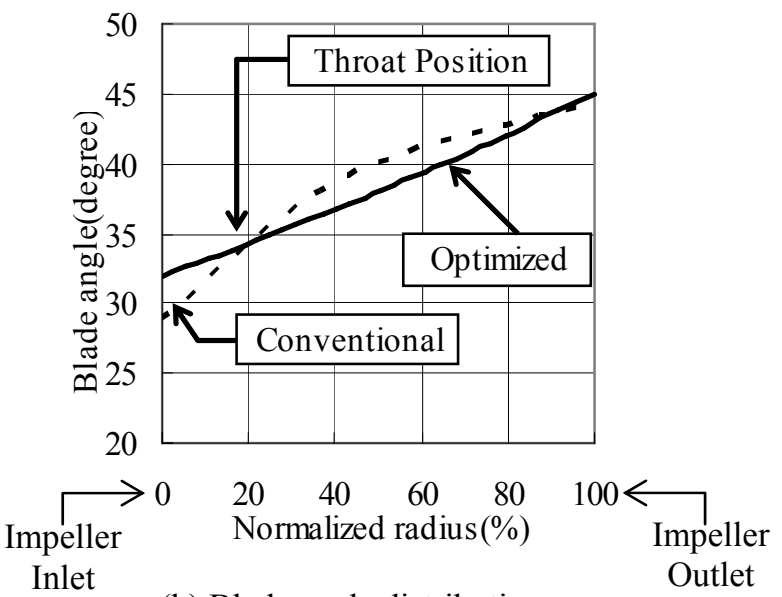

(b) Blade angle distribution

Fig. 2 Comparisons of impeller shapes

As shown in Table $1, w_{1} / w_{2}$ and $i_{1}$ increased in the optimized impeller, and the optimized impeller efficiency, $\eta_{i m p, o p t}$, increased by $1.1 \%$ over that of the conventional impeller. Moreover, $\Lambda$ increased in the optimized impeller, and the stator efficiency also increased by $1.0 \%$ in the blower stage with the optimized impeller. On the other hand, $w_{l} / w_{t h r}$ in the optimized impeller was almost the same as the conventional impeller. As depicted in Fig. 2(a), the blade height at the throat was smaller in the optimized impeller than in the conventional impeller. In addition, as shown in Fig. 2(b), the slope of the blade angle distribution around the inlet of the optimized impeller was smaller than that of the conventional impeller. Therefore, it was thought that an increase in the throat area, $A_{t h r}$, was prevented, and $w_{l} / w_{t h r}$ in the optimized impeller became almost the same as the conventional impeller. Therefore, the optimized impeller is expected to achieve at least the same stall margin as the conventional impeller.

\subsection{Diffuser Design}

In general, there is a trade-off between the efficiency at the design flow rate and the stall margin with low-solidity vaned diffusers (LSVDs). Therefore, it is important to balance the trade-off relationship in the LSVD design. Many studies have been conducted to clarify the design parameters that strongly influence the efficiency and the stall margin of the LSVD (Prasad, et al. [6], etc). These studies have shown that the incidence at the design flow rate, $i_{3}$, the turning angle in the LSVD, $\Delta \alpha$, and the solidity of the diffuser vane, $\sigma$, were the main factors that had a large influence on this trade-off relationship.

We conducted a sensitivity analysis of the efficiency at the design flow rate of the LSVD utilizing an L18 orthogonal array in order to optimize the design variables of the LSVD. Table 2 lists eight design variables in the L18 orthogonal array. As listed in the table, $i_{3}, \Delta \alpha, \sigma$ and the other variables shown in Fig. 3 were included. Moreover, diffuser-vane shapes were changed from a flat plate airfoil in the conventional diffuser to NACA 63 airfoil. Then 18 LSVDs were designed using various combinations of these design variables.

The performance of the LSVDs at the design flow rate was evaluated using three-dimensional steady flow simulation. The commercial code, ANSYS CFX 11.0 (ANSYS, Inc), was utilized for the numerical simulations. Figure 4 shows an example of the computational grid. A blade-to-blade passage from the outlet of the impeller to the outlet of a bend was modeled. The computational models and grids were made using the commercial code, ANSYS BladeGen and ANSYS TurboGrid 11.0 (ANSYS, Inc). There were about 360,000 meshes in the model. The shear Stress Transport model (Menter [7]) was used for the turbulent closure. Total pressure, total temperature, and absolute flow angle predicted for the optimized impeller were specified as the inlet boundary. The mass flow rate was specified as the outlet boundary. The efficiency reduction in the stator was derived. Total pressure losses in the downstream of the outlet boundary were taken into account by assuming the outlet component had equal $\zeta_{r c}$ to that of the conventional return channel.

Table 2 Design variables in L18 orthogonal array for diffuser

\begin{tabular}{|c|c|c|c|c|}
\hline \multirow{2}{*}{ Design variables } & \multicolumn{3}{|c|}{ Level } \\
\cline { 3 - 5 } & $Z_{\text {dif }}$ & 1 & 2 & 3 \\
\hline Number of vanes & $i_{3}$ & $-3^{\circ}$ & 13 & $0^{\circ}$ \\
\hline Incidence & $R_{3} / R_{2}$ & 1.075 & 1.100 & 1.125 \\
\hline Vane inlet radius ratio & $\sigma$ & 0.7 & 0.8 & 0.9 \\
\hline Solidity & $\Delta a$ & $4^{\circ}$ & $6.5^{\circ}$ & $9^{\circ}$ \\
\hline Turning angle & $\rho / b_{4}$ & 0.8 & 1.0 & 1.2 \\
\hline Bend curvature & $b_{45} / b_{4}$ & 0.95 & 1.00 & 1.05 \\
\hline Passage width ratio & $b_{5} / b_{4}$ & 1.0 & & 1.4 \\
\hline
\end{tabular}




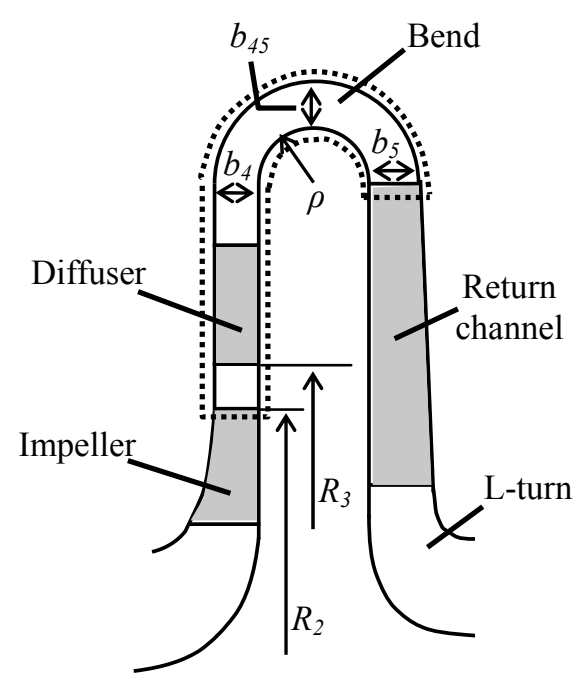

Fig. 3 Desing variables in meridional section

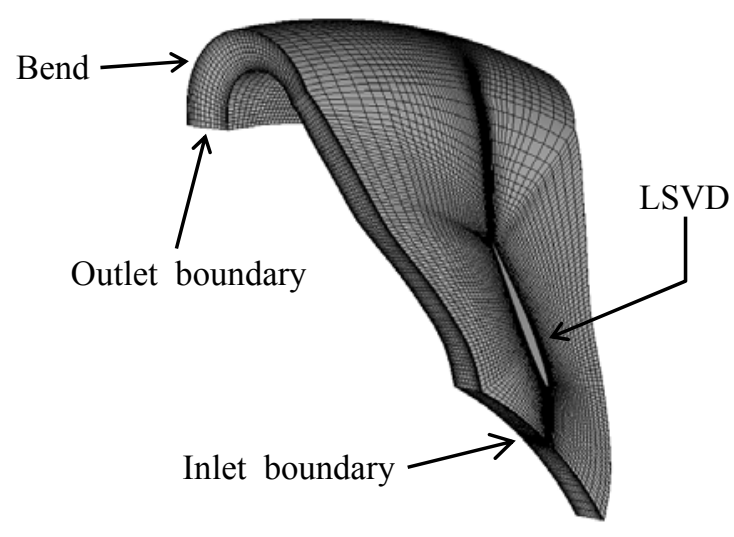

Fig. 4 Computational grid of LSVD

Figure 5 shows a cause-effect diagram of the stage-efficiency reduction. The arrows in Fig. 5 represent the value of the conventional LSVD. As shown in the figure, it is clear that $i_{3}$ and $\Delta \alpha$ have a large effect on the efficiency. However, even if the level of $i_{3}$ and $\Delta \alpha$ are set as $-3^{\circ}$ and $4^{\circ}$ to improve the stall margin of the LSVD, the stage-efficiency reduction is assumed to be about $0.6 \%$ at most. Moreover, $\sigma$ is found to have little effect on the stage efficiency. Therefore, $i_{3}, \Delta \alpha$ and $\sigma$ were set to $-3^{\circ}, 4^{\circ}$ and 0.7 , respectively. On the other hand, we can see that an increase in $b_{5} / b_{4}$ brings an improvement in the stage efficiency. However, the increase in the $b_{5} / b_{4}$ causes an increase in the axial-direction length of the blower stage. Therefore, $b_{5} / b_{4}$ was set to 1.2, which is comparable to the conventional rate. The other design variables were set to the levels indicated with circles in Fig. 5. Then, an optimized LSVD was designed with these values of the design variables.

The efficiency reduction of the optimized LSVD at the design flow rate was numerically calculated. As a result, it was confirmed that the efficiency reduction in the optimized LSVD was equivalent to that of the conventional LSVD. In addition, numerical simulations at $\phi_{l}=0.038$ (60\% of the design flow rate) were conducted for both LSVDs. Figure 6 shows the streamline in both LSVDs. As shown in Fig. 6, while a separation region was found in the conventional LSVD, it was not apparent in the optimized LSVD. Therefore, we concluded that the stall margin of the optimized LSVD improved more than that of the conventional LSVD.

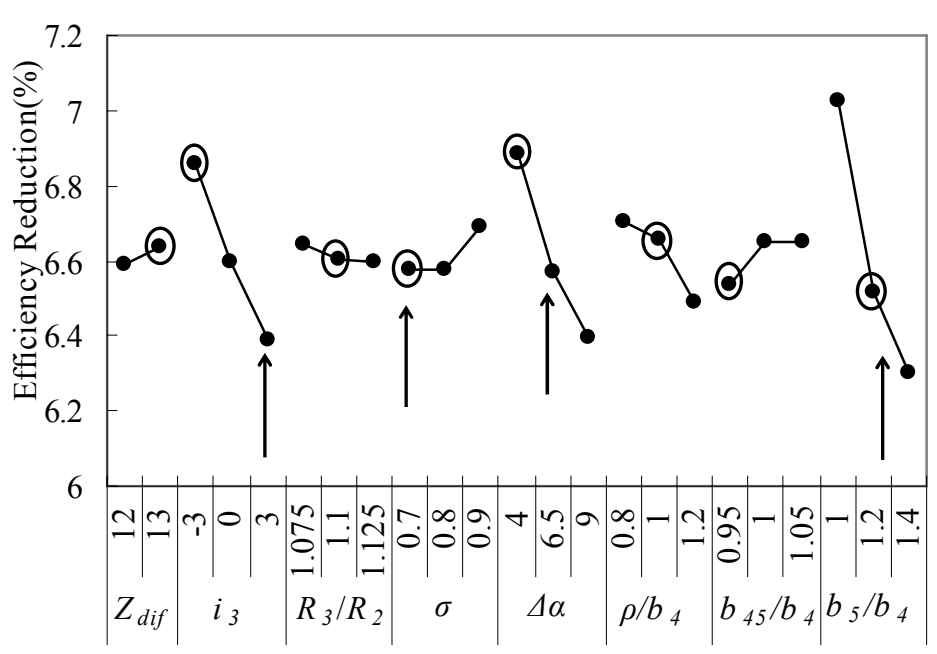

Fig. 5 Cause-effect diagram derived from calculation results of LSVD
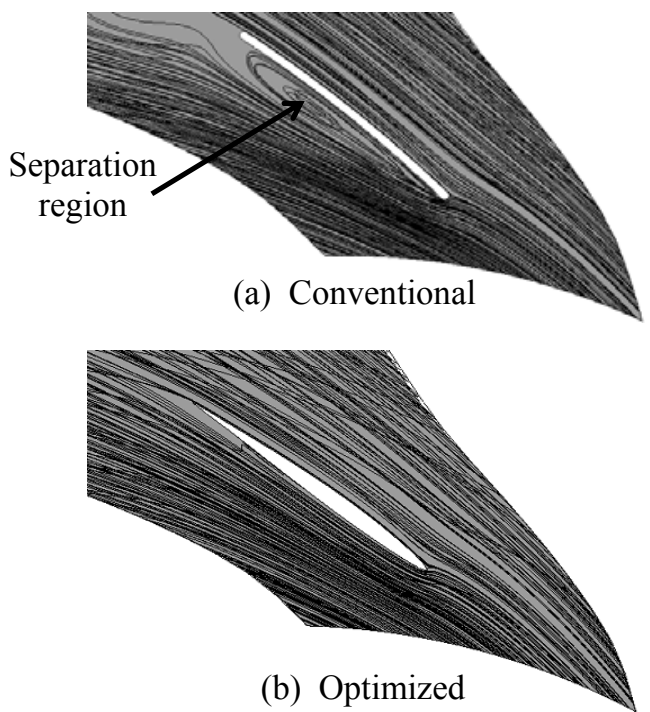

Fig. 6 Comparison of streamline in LSVD at $60 \%$ of design flow rate

\subsection{Return Channel Design}

In the new return channel, vane shapes were changed from flat plates in the conventional return channel to the NACA63 airfoil. In addition, a sensitivity analysis was conducted to evaluate the efficiency reduction of the return channel.

A preliminary flow simulation was conducted for the original return channel. The predicted value of incidence in the original return vane was $2^{\circ}$. In addition, a flow separation was found in the suction surface of the original return vane at the design flow rate. Therefore, in the new return vane, incidence, $i_{5}$, was first fixed to $-2^{\circ}$ to avoid the flow separation at the design flow rate and to improve the performance in the low-flow-rate region.

We set the number of vanes, $Z_{r c}$, an open angle, $\gamma$, a ratio of the outlet area to the inlet area of the return channel, $A_{6} / A_{5}$, and a ratio of the outlet radius to that of the conventional return channel, $R_{6} / R_{6, c v t}$, as the design variables, and utilized an L9 orthogonal array. Here, $\gamma$ is indicated in Figure 7. Table 3 gives design factors and levels utilized in the return channel. 
Table 3 Design variables in L9 orthogonal array for return channel

\begin{tabular}{|c|c|c|c|c|}
\hline \multirow{2}{*}{ Design variables } & \multicolumn{3}{|c|}{ Level } \\
\cline { 3 - 5 } & $Z_{r c}$ & 1 & 2 & 3 \\
\hline Number of vanes & $\gamma$ & 12 & 14 & 16 \\
\hline Open angle & $A_{6} / A_{5}$ & 0.6 & $17.5^{\circ}$ & $20.0^{\circ}$ \\
\hline Area ratio & $R_{6} / R_{6, c v t}$ & 1.00 & 0.7 & 0.8 \\
\hline Outlet radius ratio & & 1.05 & 1.10 \\
\hline
\end{tabular}

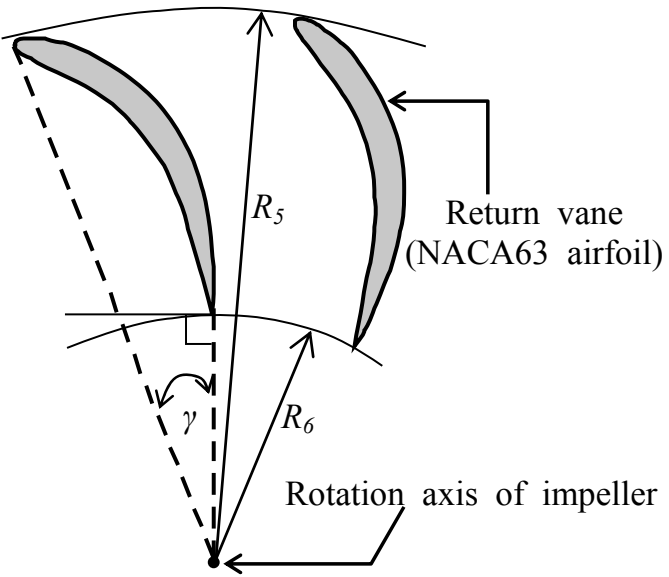

Fig. 7 Design variables in return channel

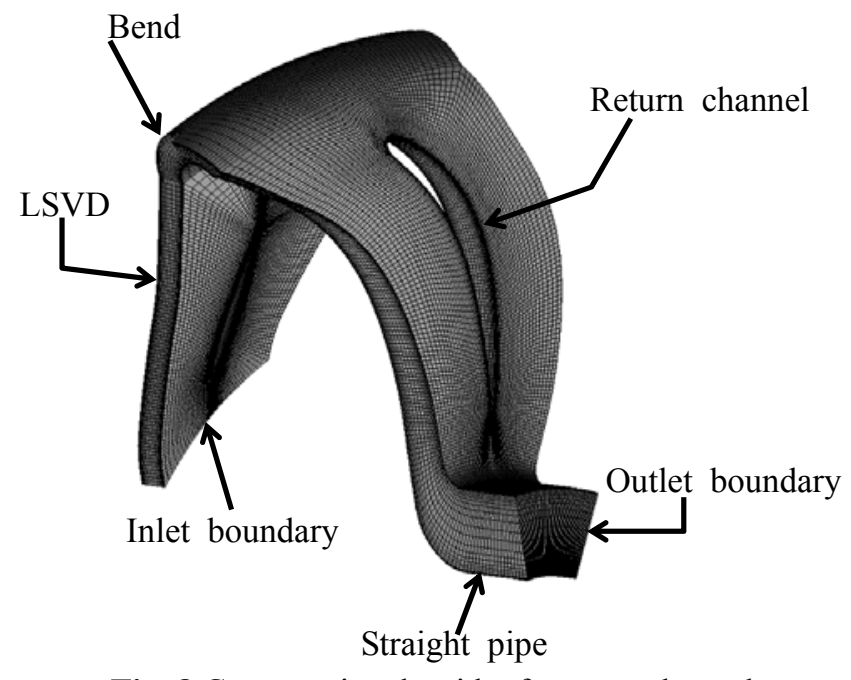

Fig. 8 Computational grid of return channel

We evaluated the performance of the return channel at the design flow rate using three-dimensional steady flow simulation. The simulation method was the same as that used in the LSVD design. Figure 8 illustrates an example of the computational grid. The computational models were made up of the optimized LSVD and the return channels. These models were connected at the center of the bend. Both domains were modeled as blade-to-blade passages. There were about 360,000 meshes in the LSVD and 280,000 in the return channels. Connections between both domains were made using a stage interface, which transferred a circumferentially averaged value at the interfaces. The efficiency reduction in the stator was then derived.

Figure 9 shows the cause-effect diagram of the efficiency reduction in the stator. An optimized return channel was designed by selecting the levels circled in Fig. 9 that resulted in smallest reduction in efficiency. As shown in Fig. 9, it was clarified that $A_{6} / A_{5}$ had a significant effect on the efficiency reduction. By investigating the calculation results, we found that $A_{6} / A_{5}$ influenced the total pressure loss in the downstream of the L-turn shown in Fig. 3. Figure 10 compares the calculated results of distributions of the total pressure loss coefficient in the meridional plane. As shown in Fig. 10, a high-loss region in the downstream of the L-turn is smaller in $A_{6} / A_{5}=0.8$ than that in $A_{6} / A_{5}=0.6$. When $A_{6} / A_{5}$ is small, the area at the return channel outlet becomes small, and the velocity at the return channel outlet becomes large. Therefore, it is considered that the flow cannot head along the outer wall at the L-turn and the separation occurs there when $A_{6} / A_{5}$ is small.

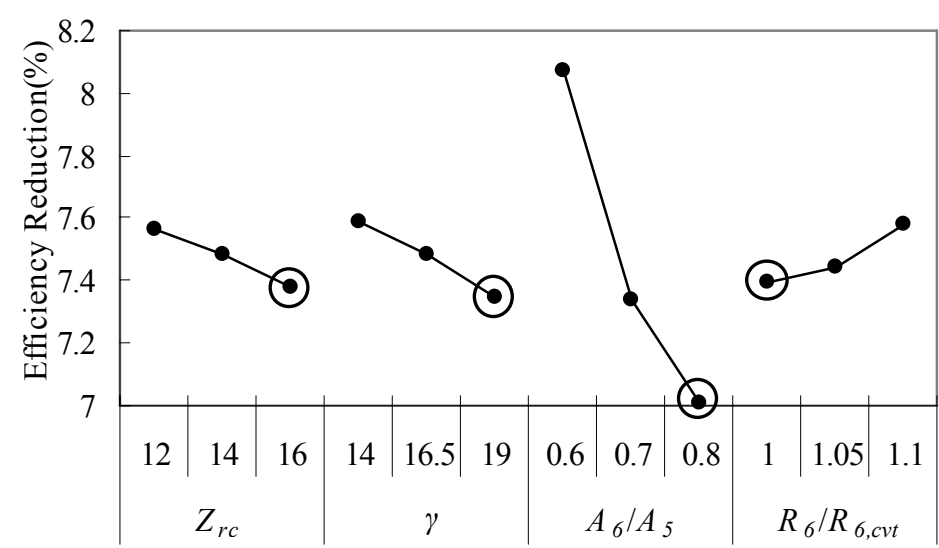

Fig. 9 Cause-effect diagram derived from calculation results of return channel
Total pressure loss coefficient

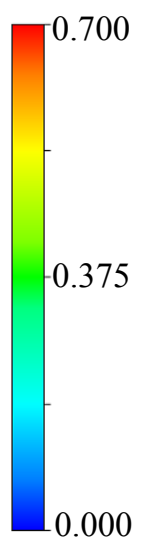

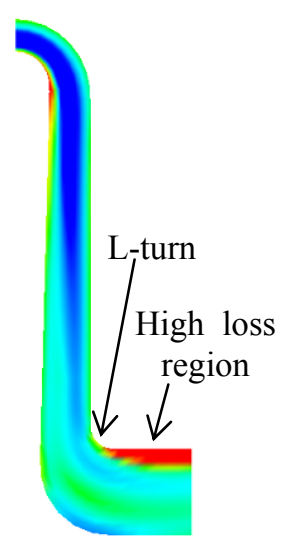

(a) $A_{6} / A_{5}=0.6$

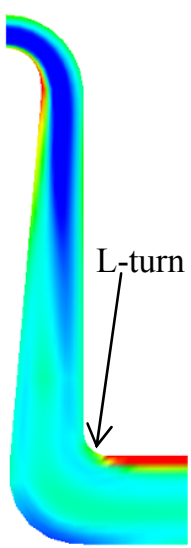

(b) $A_{6} / A_{5}=0.8$
Fig. 10 Calculation results of distributions of total Pressure loss coefficient in meridional plane 


\section{Performance Prediction and Model Test}

A performance evaluation of the optimized blower stage was conducted using the three-dimensional steady flow simulation. The method and software used in the simulation were the same as in the calculations conducted in the previous section. Figure 11 shows an example of the computational grid. The impeller with a radial suction port, LSVD, and return channel was modeled. There were about 340,000 meshes in the radial suction port and impeller. Computational models of the LSVD and return channel were the same as mentioned in the above section. Figure 12 shows a comparison of the calculated results of the stage efficiency and $\Psi$ between the conventional and optimized blower stages. As shown in the figure, the optimized blower stage improved in stage efficiency by $4 \%$ compared with the conventional stage. Moreover, $\Psi$ in the low flow rate region improved compared with the conventional blower stage. Therefore, we conducted model tests next.

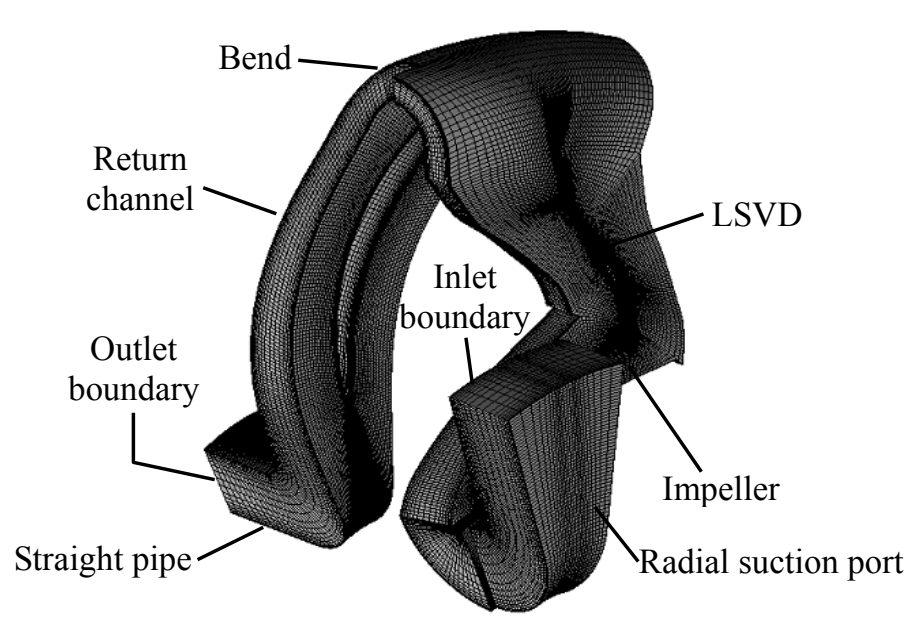

Fig. 11 Computational grid of optimized blower stage

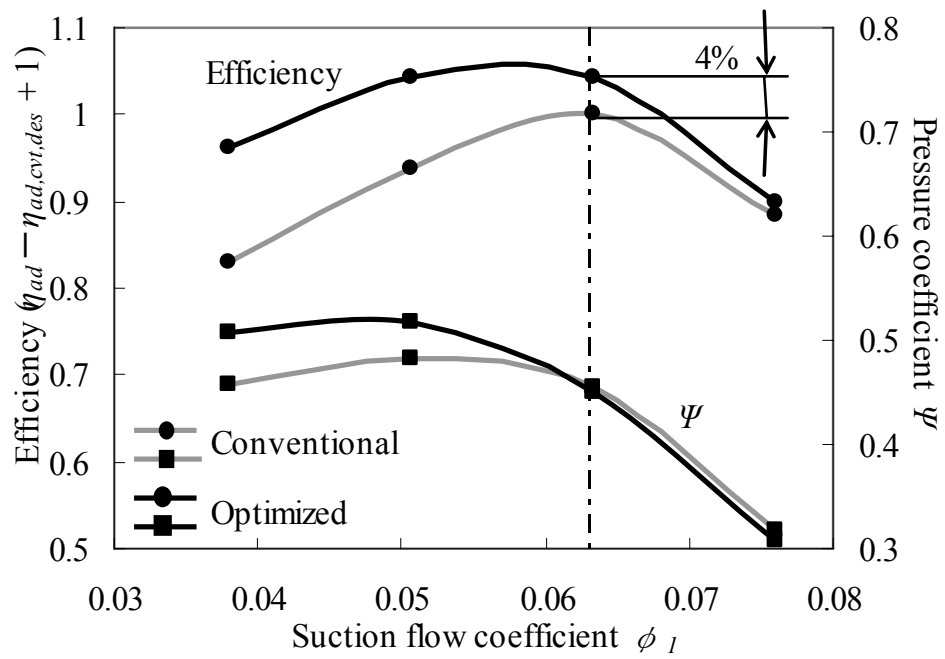

Fig. 12 Comparison of results of model calculations

In the model tests, the diameter of the impeller outlet was set to $400 \mathrm{~mm}$, and each component was designed to be similar to that designed in the previous sections. The rotational speed of the impeller was set at $6600 \mathrm{~min}^{-1}$. Figure 13 shows a comparison of the measurement result of the stage efficiency with the radial suction port in the upstream of the impeller. As shown in Fig. 13, the optimized blower stage improved in stage efficiency by $3.0 \%$ compared with the conventional blower stage. From a comparison between Fig. 12 and Fig. 13, it was clarified that the stage efficiency predicted numerically agreed well with the experimental result. In addition, a test of the surge line of each blower stage with the suction nozzle and the inlet guide vanes was conducted. This test was conducted assuming these blower stages were utilized as a first stage of the blower. A comparison of the surge line is also plotted in Fig. 13. As shown, the surge line of the optimized blower stage moved by 5.0\% toward the low-flow-rate region compared with that of the conventional one.

We concluded from our test results that energy consumption in the sewage treatment plants could be reduced using the developed blower stage in this study.

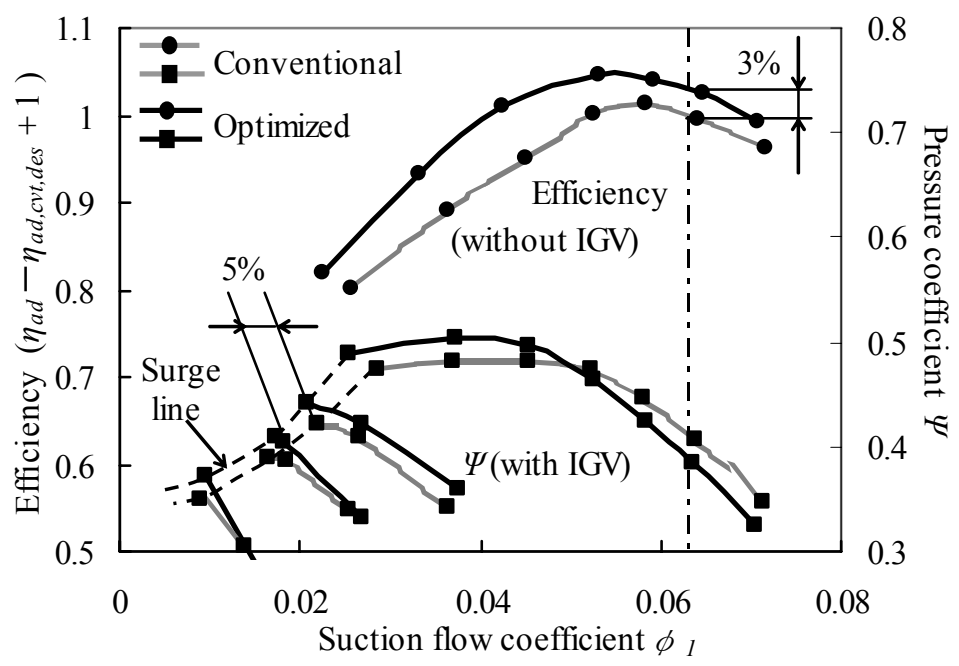

Fig. 13 Comparison of results of model tests 


\section{Conclusion}

We developed a high-performance centrifugal blower stage at design flow-rate $\phi_{1, d e s}=0.063$ to meet the demand for lower total energy consumption in sewage treatment plants. First, we optimized the two-dimensional impeller utilizing a shape optimization tool and one-dimensional performance prediction tool. A limit to the throat deceleration ratio was set in order to maintain the stall margin of the impeller. From a result of an impeller optimization, we found that stage efficiency increased by about $2 \%$ compared with the conventional blower stage. Next, a low solidity vaned diffuser was designed by using the sensitivity analysis for stage efficiency. An L18 orthogonal array was used for the sensitivity analysis. The levels of the main design variables were set in order to improve the performance in the low-flow-rate region. We confirmed that the stall margin of the optimized LSVD improved from calculated results. Moreover, the return channel was designed by using the sensitivity analysis for the total pressure loss coefficient in the return channel. An L9 orthogonal array was used for the sensitivity analysis. The levels of the design variables were set so that the total pressure loss in the return channel was minimized. Finally, model tests of both the conventional and the optimized blower stages were carried out, and the stage efficiency and operating range of both stages were compared. The optimized blower stage improved in stage efficiency by $3 \%$ and in operating range by $5 \%$ compared with the conventional blower stage. The blower stage developed in this study can thus reduce the energy consumption in sewage treatment plants.

\section{Nomenclature}

\begin{tabular}{|c|c|c|c|}
\hline$A$ & Area & $\Lambda$ & Degree of reaction \\
\hline$D$ & Diameter & & $\left(=1-\left(\rho_{2} c_{2}^{2}-\rho_{1} c_{1}^{2}\right) /\left(2 H_{t h}\right)\right)$ \\
\hline$H$ & Head & $\Psi$ & Adiabatic head coefficient \\
\hline$Q$ & Inlet volume flow & & $\left(=H_{a d} / u_{2}^{2} / \mathrm{g}\right)$ \\
\hline$R$ & Radius & $\alpha$ & Absolute flow angle \\
\hline$Z$ & Number of blades or vanes & $\beta$ & Blade angle from tangential direction \\
\hline$b$ & Channel height & $\phi$ & Flow coefficient \\
\hline$c$ & Absolute velocity & & $\left(=4 Q /\left(\pi D_{2}^{2} u^{2}\right)\right.$ \\
\hline$g$ & Gravitational acceleration & $\gamma$ & Open angle \\
\hline$i$ & Incidence $(=\beta-\alpha)$ & $\eta$ & Efficiency \\
\hline$u$ & Peripheral velocity & $\rho$ & Density or bend curvature \\
\hline$w$ & Relative velocity & $\zeta$ & Total pressure loss coefficient \\
\hline Subs & & & \\
\hline$a d$ & Adiabatic condition & $t h r$ & Throat \\
\hline$b$ & Blade & 1 & Suction or impeller inlet \\
\hline$c v t$ & Conventional & 2 & Impeller outlet \\
\hline des & Design flow rate & 3 & Diffuser vane inlet \\
\hline dif & Diffuser & 4 & Diffuser vane outlet \\
\hline imp & Impeller & 45 & Center of bend downstream of diffuser \\
\hline opt & Optimized & 5 & Return channel inlet \\
\hline rc & Return channel & 6 & Return channel outlet \\
\hline th & Theoretical & & \\
\hline
\end{tabular}

\section{References}

[1] Senoo, Y., Hayami, H., and UEKI, H., 1983, "Low Solidity Tandem Cascade Diffusers for Wide Flow Range Centrifugal Blowers," ASME Paper, 83-GT-3.

[2] Hayami, H., Senoo , Y., and Utsunomiya, K., 1990, "Application of a Low Solidity Cascade Diffuser to Transonic Centrifugal Compressor,” ASME Journal of Turbomachinery, Vol. 112, pp. 25-29.

[3] Sakaguchi, D., Ishida, M., and Ueki, H., 2008, "Analysis of Noise Generated by Low Solidity Cascade Diffuser in a Centrifugal Blower," ASME Paper, GT2008-50750, pp. 1-9.

[4] Tanaka, M., Kobayashi, H., and Nishida, H., 2009, "Development of Low Specific Speed Impellers for Centrifugal Compressors," Turbomachinery (Journal of Turbomachinery Society of Japan), Vol. 37, pp. 365-372 (in Japanese).

[5] Mishina, H., and Nishida, H., 1983, "Performance Prediction Method for Centrifugal Compressors," JSME-B, Vol. 49, No. 441, pp. 1000-1009 (in Japanese).

[6] Mukkavilli, P, Rama Raju., G., Dasgupta, A., Ramana Murty, G.V., and Jagadeshwar Chary, K.V., 2002, "Flow Studies on A Centrifugal Compressor Stage with Low Solidity Diffuser Vanes," ASME Paper, GT2002-30386, pp. 1-9..

[7] Menter, F. R., 1994, "Two-Equation Eddy-Viscosity Turbulence Models for Engineering Applications," AIAA-Journal, Vol. 32, No. 5, pp. 1598-1605. 